\title{
Assessment of Laboratory Safety in Universities Based on Combined Weighting Method and Unascertained Measure Theory
}

\author{
Kong Shengli ${ }^{1}$, Song Yuqing ${ }^{1}$, Yang Pengfei ${ }^{1}$, Fang Xinmei ${ }^{1}$ Yang Zhen ${ }^{1}$, Tang Chenlu ${ }^{1}$ and Wang Wei ${ }^{2, *}$ \\ ${ }^{1}$ School of Urban Construction and Safety Engineering, Shanghai Institute of Technology, Shanghai 201418, China \\ ${ }^{2}$ Shanghai Fire Science and Technology Research Institute of MEM, Shanghai 200032, China
}

\begin{abstract}
In order to avoid accidents and improve the level of laboratory safety management in universities, a laboratory safety assessment method based on entropy weight method, analytic hierarchy process and unascertained measure theory is put forward. The laboratory safety assessment index system is established by selecting eight assessment indexes such as hazardous chemicals management, instrument and equipment management and personal protective equipment management; The comprehensive weight of the assessment index is determined by combining the combination weighting method of analytic hierarchy process (AHP) and entropy weight method (EW). According to the principle of maximum membership degree, the safety grade of university laboratory is determined, and compared with the actual results. This work indicated that this innovated method can reasonably evaluate the current situation of laboratory safety, and it can provide a reference for strengthening laboratory safety management.
\end{abstract}

\section{Introduction}

Accidents, in recent years, occurred frequently in numerous college laboratories and caused significant losses and negative social influences[1]. Although a number of hazardous materials in university laboratories is less than industry, safety problems are always difficult to properly solve due to the high mobility of personnel, insufficient individual experience and incomplete safety management system.

Several scholars have established mathematical models for safety assessment by introducing mathematical theories such as fuzzy mathematics[2], error back propagation (BP) neural network[3], gray correlation[4] and set pair analysis[5]. Simultaneously, good results have been achieved in research and establishment of the laboratory safety assessment system[6]. This study consulted related materials, then found that they are related to the safety of university laboratories.

The comprehensive weight of the assessment index is determined by coupling the subjective weighting method Analytic Hierarchy Process (AHP) and the objective weighting method Entropy Weighting (EW). At present, the laboratory safety level assessment of colleges usually adopts a checklist scoring method, but the scoring information in the checklist is mostly uncertain. Based on Wang[7], it can appropriately solve the problem of information uncertainty[8-11]. Accordingly, this study can adopt EW-AHP and unascertained measurement theory and assessment index system to enrich the safety assessment theory and provide a reference for improving the safety level of universities laboratories.

\section{EW-AHP and Unascertained Measure Theory}

Assuming that the index space $A=\left\{a_{1}, a_{2}, a_{3}, \ldots, a_{n}\right\}$ has $n$ elements, and $H=\left\{h_{1}, h_{2}, h_{3}, \ldots, h_{m}\right\}$ is the $m$ assessment index for each element, an $m$-dimensional vector of $n a_{i}=\left\{a_{i 1}, a_{i 2}, a_{i 3}, \ldots, a_{i m}\right\}$ is formed, and $a_{i j}$ represents the assessment index measurement value of the laboratory safety level. Divide $a_{i}$ into $s$ assessment levels, mark the assessment level $U=\left\{D_{1}, D_{2}, D_{3}, \ldots, D_{s}\right\}$, and set it as $D_{e}>D_{e+l}$, then call $\left\{D_{1}, D_{2}, D_{3}, \ldots, D_{s}\right\}$ an ordered segmentation class on the space $U[12-13]$.

\subsection{Single index unascertained measure}

If $u_{i j e}=u\left(a_{i j} \in D_{e}\right)$ represents the degree to which the measurement value $a_{i j}$ belongs to the $e$ assessment level $D_{e}$, and requires $u$ to satisfy "non-negativity", "normalization" and "additivity". $D$ needs to satisfy the following Formula (1)-(3).

$$
\begin{gathered}
0 \leq u\left(a_{i j} \in D_{e}\right) \leq 1 \\
u\left(a_{i j} \in U\right)=1 \\
u\left(a_{i j} \in \bigcup_{l=1}^{e} D_{l}\right)=\sum_{l=1}^{e} u\left(a_{i j} \in D_{l}\right) \\
(i=1,2, \cdots, n ; j=1,2, \cdots, m ; l=1,2, \cdots, e)
\end{gathered}
$$

According to the definition of the unascertained measure, $u$ that satisfies the above formula is called the unascertained measure, and the matrix $\left(u_{i j e}\right)_{m \times s}$ is constructed as the single-index measurement assessment matrix as follows: 


$$
\left(u_{i j e}\right)_{m \times s}=\left[\begin{array}{cccc}
u_{i 11} & u_{i 12} & \cdots & u_{i 1 s} \\
u_{i 21} & u_{i 22} & \cdots & u_{i 2 s} \\
\vdots & \vdots & \ddots & \vdots \\
u_{i m 1} & u_{i m 2} & \cdots & u_{i m s}
\end{array}\right]
$$

The Formula (4) displays the single index measurement assessment matrix.

\subsection{EW-AHP determines the weight coefficient}

\subsubsection{Entropy Weight Method (EW)}

The entropy weighting method (EW) is an objective weighting method that via entropy $v_{i j}$ to measure the amount of information and then determine the importance of assessment indicators. Assuming that $h_{j}$ is used to characterized the importance of an indicator $\theta_{i j}$

$\left(0 \leq \theta_{i j} \leq 1, \quad \sum_{j=1}^{m} \theta_{i j}=1\right) \quad$ to all other indicators, then $\theta_{i j}$ is the entropy weight value of indicator and the entropy weight values of other indicators $h_{j}$ can be obtained in the same way. The calculation Formula (5)-(6) as follows:

$$
\begin{aligned}
& v_{i j}=1+\frac{1}{\lg s} \sum_{i=1}^{s} u_{i j e} \lg u_{i j e} \\
& \theta_{i j}=v_{i j} / \sum_{i=1}^{n} v_{i j}
\end{aligned}
$$

\subsection{Analytic hierarchy process (AHP)}

The analytic hierarchy process (AHP) is a subjective weighting method. First, the factors that affect the safety level of the laboratory are divided into levels and pairwise comparisons which are made according to the 9-level scale method to construct the judgment matrix $S$. Then use the square root method to calculate the judgment matrix to get the weight value of each factor.

Among them, it is greatly necessary to check the consistency of the judgment matrix $S$. When it is determined that the random consistency ratio (CR) is less than 0.1 and it is confirmed that the result of the analytic hierarchy process meets the requirements. Otherwise, each value in the judgment matrix needs to be adjusted, recalculated and verified.

\subsubsection{EW-AHP combination empowerment}

This work applied the multiplicative synthesis normalization method to calculate the comprehensive weight of each indicator[14], the calculation formula as follows:

$$
\omega_{j}=\left(\alpha_{j} \cdot \beta_{j}\right) / \sum_{j=1}^{m}\left(\alpha_{j} \cdot \beta_{j}\right)
$$

(Among then, $\alpha_{j}$ and $\beta_{j}$ are the subjective and objective weights of the $j$ assessment index respectively, $\omega_{j}$ is the comprehensive weight of the $j$ assessment index, and $m$ is the number of assessment index.)

\subsection{Multi-index comprehensive measure}

The calculation formula for the multi-index measure obtained by combining the comprehensive weight as follows:

$$
u_{i e}=\sum_{j=1}^{m} \omega_{i j} u_{i j e}
$$

The calculation result still satisfies $0 \leq u_{e} \leq 1, \sum_{e=l}^{s}$ and $u_{i e}=1$, then $u_{i e}$ is also an unascertained measure and $u_{i e}=$

$\left(u_{i 1}, \quad u_{i 2}, u_{i 3}, \ldots, u_{i s}\right)$ is obtained, which is recorded as the multi-index comprehensive measure assessment vector.

\section{Case application and analysis}

\subsection{Survey of Research Objects}

The research object is a laboratory affiliated to a secondary college of a Shanghai university. Based on the system of each secondary college of the university in charge of the laboratory to which it belongs and taking into account the safety management mode of the laboratory of different colleges and the difference in the nature of teaching or scientific research. Among them, during the use of laboratories such as the School of Humanities and the School of Economics and Management, laboratories with low risk of occurrence are not within the scope. This study only focuses on the laboratories of six colleges with relatively high risk and numbered the six colleges as B1, B2, B3, B4, B5 and B6.

\subsection{Selection and classification of assessment indicators}

The domestic university laboratory safety assessment system established is shown in Figure 1. below, which includes the follow laboratory safety ing 8 main assessment indicators[15-19]: Hazardous chemicals management (X1). Instrument and equipment management (X2). Personal protective equipment management (X3). Laboratory waste disposal (X4). Emergency plan and facilities (X5). Personnel training management (X6). Safety culture (X7). Laboratory access system (X8).

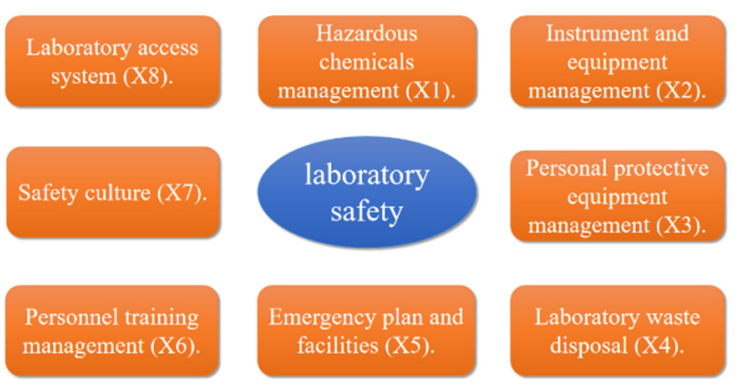

Figure 1. Safety assessment index system

In this case, the laboratory safety level is divided into: $U=\left\{D_{1}, D_{2}, D_{3}, D_{4}, D_{5}\right\}=\{$ very unsafe, unsafe, generally safe, safe, very safe $\}$. The eight first-level assessment indicators in the laboratory safety assessment system are 
scored through quarterly and annual inspection score sheets. The scoring standards are mainly based on the completion of the work content described in the corresponding second-level assessment indicators. The scoring uses a hundred-point system, and the division is in accordance with: $0 \leq D_{1}<50,50 \leq D_{2}<60,60 \leq D_{3}<75$, $75 \leq D_{4}<90,90 \leq D_{5} \leq 100$ 。

\subsection{Construct single index function}

Its laboratories seldom use chemicals, and most of them use various instruments and equipment taking the B3 laboratory as an example. For other types of accidents, the waste generated by the experiment is usually metal, wood and other wastes, and most of them are non-hazardous. In the process of managing laboratories, the college prepares emergency rescue plans for each. To forming a better safety culture, it is equipped with enough emergency facilities have been adequately studied, trained and assessed for personnel who related to laboratory.

By constructing a single-index measurement function $u$, the value $u_{i k}$ of each unascertained measurement can be obtained directly. According to the unascertained measure definition, the single index measure function of the assessment model is constructed as follows:

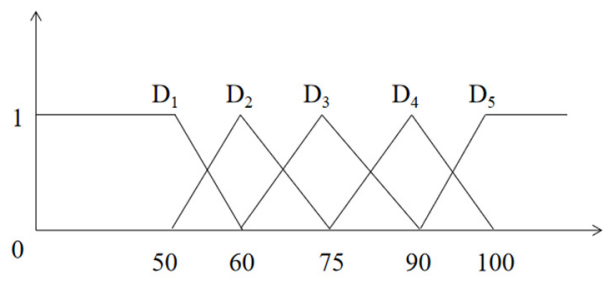

Figure 2. Single index measurement function of each assessment index

Table 1. Laboratory safety management status statistic table

\begin{tabular}{|c|c|c|c|c|c|c|c|c|}
\hline No. & X1 & X2 & $\mathbf{X 3}$ & $\mathbf{X 4}$ & $\mathbf{X 5}$ & $\mathbf{X 6}$ & $\mathbf{X 7}$ & $\mathbf{X 8}$ \\
\hline B1 & 70 & 85 & 80 & 75 & 80 & 85 & 85 & 85 \\
\hline B2 & 70 & 70 & 75 & 80 & 95 & 85 & 80 & 90 \\
\hline B3 & 65 & 65 & 80 & 80 & 85 & 90 & 90 & 90 \\
\hline B4 & 80 & 80 & 90 & 80 & 90 & 90 & 90 & 90 \\
\hline B5 & 75 & 80 & 80 & 90 & 80 & 85 & 80 & 85 \\
\hline B6 & 75 & 70 & 80 & 80 & 90 & 90 & 80 & 95 \\
\hline
\end{tabular}

From Table 1, the assessment matrix of B2 single index measurement can be obtained as shown in Figure 2.

$$
\left(u_{4 j e}\right)_{8 \times 5}=\left[\begin{array}{ccccc}
0 & 0.667 & 0.333 & 0 & 0 \\
0 & 0.667 & 0.333 & 0 & 0 \\
0 & 0 & 0.667 & 0.333 & 0 \\
0 & 0 & 0.667 & 0.333 & 0 \\
0 & 0 & 0.333 & 0.667 & 0 \\
0 & 0 & 0 & 1 & 0 \\
0 & 0 & 0 & 1 & 0 \\
0 & 0 & 0 & 1 & 0
\end{array}\right]
$$

\subsection{EW-AHP combined weighting to determine the weight of assessment index}

Combined the AHP and EW methods and employed Formula (7) to calculate the comprehensive weight of each assessment index. Taking B3 as an example, the specific calculation process as follows:

1) The AHP method determines the weight. According to the 9-level scale method in the AHP method, the 8 indicators are used to construct a judgment matrix by comparing the importance of each factor through expert assessment and through the consistency test, the subjective weight of each assessment indicator is finally obtained, as shown in Table 2.

2) The EW method determines the weight. The amount of information provided by the assessment index is determined according to the degree of variation of the index, which can directly reflect the effect of a certain assessment index in all indexes. Therefore, this method can be used as an objective assessment index weight calculation method in comprehensive assessment. According to the Formula (5)-(6), the objective weight of each assessment index can be calculated as Table 2 .

3) The combination weighting method determines the weight. Using the multiplicative normalization method, combining the subjective weight of the assessment index with the objective weight according to Formula (7), the comprehensive weight of each assessment index can be calculated as shown in Table 2.

Table 2. Comprehensive weight value of assessment index of laboratory safety management level

\begin{tabular}{|c|c|c|c|}
\hline $\begin{array}{c}\text { Assessment } \\
\text { indexes }\end{array}$ & AHP & EW & AHP-EW \\
\hline $\mathrm{X} 1$ & 0.2568 & 0.1531 & 0.3620 \\
\hline $\mathrm{X} 2$ & 0.0424 & 0.3123 & 0.1219 \\
\hline $\mathrm{X} 3$ & 0.1228 & 0.1062 & 0.1201 \\
\hline $\mathrm{X} 4$ & 0.0841 & 0.1062 & 0.0822 \\
\hline $\mathrm{X} 5$ & 0.1165 & 0.1426 & 0.1530 \\
\hline $\mathrm{X} 6$ & 0.2056 & 0.0286 & 0.0542 \\
\hline $\mathrm{X} 7$ & 0.0564 & 0.0991 & 0.0515 \\
\hline $\mathrm{X} 8$ & 0.1154 & 0.0519 & 0.0551 \\
\hline
\end{tabular}

\subsection{Determination of Assessment Vector of Multi-index Comprehensive Measure}

According to Formula (8), the comprehensive weight of B3 laboratory safety assessment index and its single index measurement assessment matrix, then the multi-index comprehensive measurement vector $\boldsymbol{u}_{3 e}=\left(\begin{array}{ll}0 & 0 \text {. }\end{array}\right.$ 32280.34700 .33020 ) of B3 can be obtained and according to the principle of maximum membership degree, its safety level is general safety. Using the same method, the multi-index comprehensive assessment vector and safety level of the data samples of the other colleges' laboratory can be obtained. The final assessment results are compared with the actual assessment results in the following Table 3. 
Table 3. The Assessment Results of Unascertained Measure Theory Based on EW-AHP

\begin{tabular}{|c|c|c|c|c|c|c|}
\hline \multirow[b]{2}{*}{ No. } & \multicolumn{5}{|c|}{ Comprehensive Unascertained Measure } & \multirow{2}{*}{$\begin{array}{c}\text { Assessm } \\
\text { ent } \\
\text { Results }\end{array}$} \\
\hline & $\begin{array}{l}\text { D } \\
1\end{array}$ & D2 & D3 & D4 & D5 & \\
\hline B1 & 0 & 0.1205 & 0.600 & 0.2795 & 0 & D3 \\
\hline B2 & 0 & 0.1610 & 0.5500 & 0.2125 & 0.0765 & D3 \\
\hline B3 & 0 & 0.3228 & 0.3470 & 0.3302 & 0 & D3 \\
\hline B4 & 0 & 0 & 0.3776 & 0.6224 & 0 & D4 \\
\hline B5 & 0 & 0 & 0.6254 & 0.3746 & 0 & D3 \\
\hline B6 & 0 & 0.0406 & 0.6401 & 0.3193 & 0.0276 & D3 \\
\hline
\end{tabular}

\subsection{Analysis and Discussion}

The calculation results of the index weights in Table 2 showed that the importance of each index calculated by the subjective AHP method is exceedingly different from EW. The combined weighting method can eliminate the shortcomings of the two methods within weighting. The major influencing factors of laboratory safety management are hazardous chemical management, emergency plans and facilities, instrument and equipment management and relatively small influencing factors are laboratory access system, safety culture construction and personnel training management.

Table 3 revealed the assessment results of the laboratory safety level of the six secondary colleges of the university based on EW-AHP and unascertained measurement theory, which shows that only one is relatively safe and the rest are generally safe. Actual assessment on site that only one is relatively safe but five is generally safe, which is consistent with the actual inspection results.

The scoring results in Table 1 should be used to formulate corresponding countermeasures for laboratory safety and to adjust safety management strategies in a targeted manner to improve laboratory safety.

\section{Conclusion}

1) Comprehensively considering the eight factors that affect the safety level of college laboratories, the established college laboratory safety assessment index system can better reflect the safety status of college laboratories.

2) Due to the uncertainty of the evaluator's score, the laboratory safety assessment theory established based on the entropy weight method, the analytic hierarchy process and the unascertained measurement theory, the analysis of examples shows that the model method is reasonable and effective.

\section{Acknowledgements:}

Sponsored by National Science Foundation of China (51604174; 51706122) , Shanghai Rising-Star Program (20QB1401000), the Natural Science Foundation of Shanghai (19ZR1411500), the Science \& Technology Foundation of Guizhou Province ([2020]4Y055). "Chen Guang” project supported by Shanghai Municipal
Education Commission and Shanghai Education Development Foundation (17CG60)

\section{References}

1. Yuanxing. YE, Jing. MA, Yuze. ZHAO, Yilan. SHEN, Zhongcheng. REN, Experimental Technology and Management 37, 317 (2020)

2. Lirong. WU, Yalong. QU, Weimin. CHENG, Research and Exploration in Laboratory 39, 300 (2020)

3. Jiaming. Zhang, Rendong. Jin, Wenrui. Wang, Hao. Sun, Honggang. Yi,Wr., Research and Exploration in Laboratory 39, 290 (2020)

4. Qian. Ma, Chaoxia. Wang, Research and Exploration in Laboratory 38, 276 (2019)

5. Hao. Li, Jia. Yao Li, Research and Exploration in Laboratory 34, 285 (2015)

6. Ding. Li, Pei. Cao, Ping. Wang, Research and Exploration in Laboratory 33, 274 (2014)

7. Guangyuan. Wang, Journal of Harbin University of Civil Engineering and Architecture 23, 1 (1990)

8. Yulong. He, Bingshuo. Chen, Pengwang. Gao, Lei. Liu, Jiaxin. Chi, Journal of Chongqing Jiaotong University (Natural Science Edition) 40, 8 (2021)

9. Li. Zhang, Mingsheng. Zhao, Enan. Chi, Zhenqian. Ma, Mingqing. Zhang, Teng. Wang, China Mining Magazine 29, 147 (2020)

10. Wenlong. Li, Huimin. Li, Xingwwang. Pei, Hai. Meng, Yijun. Liu, Engineering Journal of Wuhan University 53, 1065 (2020)

11. Qiang. Zhai, Weihong. Gu, Safety and Environmental Engineering 27, 92 (2020)

12. Chenyang. Zhao, Faicheng. Chi, Xu. Peng, ISA Transactions 101, 503 (2020)

13. Yu. Si , Analytical cellular pathology (Amsterdam) 2020, 1 (2020)

14. Shuheng. Zhong, Yanjiao. Li, Coal Engineering 53, 60 (2021)

15. Fagang. Zhao, Hongwei. Cui, Yanfeng. Geng, Baochun. Li, Haitao. Zhou, Research and Exploration in Laboratory 39, 300 (2020)

16. Fengxin. Gao, Shixiang. Lu, Research and Exploration in Laboratory 33, 272 (2014)

17. Yinzhu. Zhang, Haipin. Jin, Jun. Ruan, Huanjun. Yu, Jianyue. Feng, Experimental Technology and Management 32, 226 (2015)

18. Guoqing. Cha, Yani. Xu, Yifei. Qin, Experimental Technology and Management 37, 271 (2020)

19. Lin. Zhang, Yingzi. Guo, Dongming. Xu, Yongheng. Jiang, Experimental Technology and Management 33, 227 (2016) 